\title{
Antibiotic Susceptibility Pattern of Bacterial Isolates from Patients of Respiratory Tract Infection at 43 Centers in Punjab, Pakistan
}

\section{Ali I* and Butt MA}

Department of Pharmacology, University College of Pharmacy, University of the Punjab, Lahore, Pakistan

*Corresponding author: Dr. Ali I, Department of Pharmacology, University College of Pharmacy, University of The Punjab, Lahore, Pakistan, Tel: +92 322 4660448; E-mail: ijaz_708@yahoo.com

Received date: January 27, 2017; Accepted date: February 15, 2017; Published date: February 22, 2017

Copyright: (c) 2017 Ali I, et al. This is an open-access article distributed under the terms of the Creative Commons Attribution License, which permits unrestricted use, distribution, and reproduction in any medium, provided the original author and source are credited.

\begin{abstract}
Respiratory tract infections are the most common diseases that are associated with social burden for the patient. In most cases, treatment is started before the culture sensitivity. Empiric therapy is based on symptoms and the medical practitioner's experience. Antibiotic resistance has become a serious health problem in the world, and also in Pakistan. This study was conducted to ascertain the current scenario of bacterial susceptibility in respiratory tract infection in order to optimize empiric therapy among people of all groups of ages in Punjab province located in Pakistan. Forty three (43) centers were selected randomly in major cities of Punjab. The numbers of patients investigated in these centers were 311. The study was undertaken from January 2012 to December, 2012. This period spans all seasons in the study areas. Out of 311 bacterial isolates, Klebsiella species have been isolated in 41 cases (13.06\%), Escherichia coli $74(23.57 \%)$, Acinetobacter 43 (13.69\%), $\beta$-hemolytic streptococcus 15 (4.78\%), Pseudomonas species 111 (35.35\%), Streptococcus pneumonia $10(3.18 \%)$ and Staphylococcus aureus in 17 cases $(5.41 \%)$. Gram-positive bacteria were isolated from 42 patients $(13.51 \%)$ while Gram-negative bacteria were isolated from 269 patients $(86.49 \%)$. On an average 30 antibiotics of various classes have been tested for the susceptibility of gram-positive bacteria and gram-negative bacteria. $66.25 \%$ of the antibiotics tested showed susceptibility to the gram-positive bacteria. Vancomycin showed maximum sensitivity $(100 \%)$ to gram-positive bacteria followed by Linezolid $(97.44 \%)$ and Fusidic acid $(83.34 \%)$. On the other hand $33.44 \%$ of the antibiotics tested showed sensitivity to the gram-negative bacteria. Cefoperazone/Sulbactam showed maximum sensitivity $(91.39 \%)$ to gram-negative bacteria followed by Imipenem $(72.75 \%)$ and Pipercillin/Tazobactam $(71.60 \%)$.
\end{abstract}

Keywords: Respiratory tract; Antibiotics; Pneumonia; Bronchitis; Drug side effects

\section{Introduction}

Respiratory infections occur more often than they are reported, and are responsible for more duration of bed disability, restricted activity, stay away from job and school than the other reported acute illnesses. Respiratory tract infections account for more than $40 \%$ of days of disability secondary to acute illness and pneumonia and influenza are among ten leading causes of death in general population, and $80-90 \%$ of the deaths occur in the elderly (persons $>65$ years of age) $[1,2]$. The actual number is much higher because this figure does not include persons died of pneumonia, with other conditions (e.g., HIV, alcohol and tobacco-related diseases) [3].

Among bacterial infections, respiratory tract infections range from pharyngitis and otitis media to acute bronchitis and pneumonia. Respiratory tract infections are the most common diseases that are associated with social burden for the patient. In most cases, treatment is started before the culture sensitivity.

Financial influence of respiratory infections in 1999 was \$25.6 billion [4]. These figures largely underestimate the economic impact of outpatient treatment of respiratory tract infections. Statistics reported by the National Health Interview Survey evaluate that 182 million incidents of respiratory tract infection occurs in which no medical attention is solved. Often, individuals with respiratory tract infections try over-the-counter medicines for relief of their symptoms, and only seek medical treatment when these efforts become useless. OTC medications in population, contribute towards the $\$ 456,000,000$ annually to treat respiratory infections $[3,4]$.

In Pakistan since July 29, 2010 to December 22, 2011, approximately $12,887,440$ of acute respiratory infection cases are reported. Patient consultations have been reported in DEWS (Disease Early Warning System) from the flood affected districts four provinces in Pakistan which is about $23 \%$ of total consultations [5] as described in following Table 1.

\begin{tabular}{|l|l|}
\hline Diseases & Total \\
\hline Acute diarrhea & $1,346,646(8 \%)$ \\
\hline Bloody diarrhea & $84,324(0.5 \%)$ \\
\hline Acute Respiratory Infection & $3,443,184(20 \%)$ \\
\hline S. Malaria & $802,606(5 \%)$ \\
\hline Skin Diseases & $775,032(4 \%)$ \\
\hline Unexplained fever & $612,683(3 \%)$ \\
\hline Total (All consultations) & $17,584,383$ \\
\hline
\end{tabular}

Table 1: Respiratory infection cases.

Antibiotic resistance has become a serious health problem in the world, and also in Pakistan. The United States Centre for Disease 
Prevention and Control (CDC) calls antibiotic resistance one of its "top concerns". This is because over the last decade almost all types of bacteria have become less susceptible to antibiotic treatment when it is really needed.

Gram-negative organisms produce $\beta$-lactamases, and this is a major factor in the resistance of the semi synthetic broad-spectrum $\beta$-lactam antibiotics. Aminoglycosides are inactivated by phosphorylation, acetylation, adenylation and the necessary enzymes are found in both gram-negative and gram-positive plasmids and several on transposon. Plasmid-mediated modification of protein binding site on the $50 \mathrm{~S}$ subunit is the basis of resistance to erythromycin, fluoroquinolones and decreased binding due to a point mutation in the DNA gyrase. Plasmid- mediated resistance to tetracyclines is an important example of reduced drug accumulation; these are also found in both grampositive and gram-negative bacteria [6]. The resistance of $S$. aureus to erythromycin and other macrolides, and fluoroquinolones, is due to an energy-dependent efflux. Inhibitors such as pumps may be useful additional antibiotics [7]. Disturbing development of resistance has been found in the Staphylococci, one of the most common causes of hospital systemic infections, a number of species that are now resistant to almost all currently available antibiotics [8].

Nature has bestowed microorganism with devilishly effective adaptive mechanisms for putting down the best treatment strategies, and we try to remove them. This challenging situation has been reviewed thoroughly [9].

Dosing errors are common in antibiotics administration. Excessive dosage can cause significant side effects, while too little can lead to treatment failure, and lead to antibiotic resistance [10].

Antibiotic resistance is unavoidable and emerges mostly when a patient is given empiric therapy. This is mainly due to insufficient research work on antibiotic sensitivity patterns. The present study is an attempt to find out the current status of antibiotic sensitivity pattern of common bacterial isolates at Punjab level. The aim of this study is to optimize empiric therapy in Respiratory tract infections.

\section{Methods}

The study involved major cities of Punjab (Pakistan). Forty three (43) centers were selected randomly in major cities of Punjab. The patients of all groups of ages were included in study. The study aim and objectives were explained to all the participating patients through laboratory staff and it was assured that all the information obtained from them will be kept confidential. Based on this, consent and verbal approval granted. Similarly, the administrative heads of selected laboratory centers were assured that all information of patients will remain confidential. We therefore identified the laboratory centers in Punjab both in hospitals and in community. The numbers of patients investigated in these centers were 311 . Selected laboratory centers were asked to collect specimens from patients suffering from respiratory tract infections. Specimens were as follows: Throat swab; Sputum; Bronchial washing; Tracheal secretion.

Specimens were enclosed within specialized containers. A transport medium (Amies Transport medium) was also present within these containers. These were recapped tightly. After collection, each specimen was sent to their central laboratory for culture sensitivity test. The samples were inoculated into sheep blood agar, MacConkey agar and chocolate agar medium and incubated at $37^{\circ} \mathrm{C}$ for $48 \mathrm{~h}$.
Antimicrobial susceptibility testing was performed using disc diffusion method.

$\mathrm{BaSO}_{4}$ turbidity standard is used for inoculums which is equivalent to a $0.5 \mathrm{McF}$ arland standard or its optical equivalent (e.g., latex particle suspension). The swab is inoculated into the dried surface of a MuellerHinton agar plate by streaking it over the entire sterile surface of agar medium. The anti-microbial discs are dispensed onto the surface of the inoculated agar plates by using automated disc dispenser. Pathogens were identified after $48 \mathrm{~h}$ of incubation [11]. Selected Laboratory centers strictly follow CLSI (Clinical and Laboratory Standards Institute) previously known as NCCLS (National Committee for Clinical Laboratory).

Only positive culture sensitivity reports were selected. The data was collected on properly designed Performa and also in the form of photocopies of original reports.

\section{Statistics}

Minimal statistics (only percentage and average) is applied in Microsoft Excel 2007 in data entry and result interpretation.

\section{Results}

In total 311 respiratory pathogens were isolated in Punjab during study period of January 2012 to December 2012. Seven types of bacteria were isolated. Out of 311 bacterial isolates, Klebsiella species have been isolated in 41 cases (13.06\%), Escherichia coli 74 (23.57\%), Acinetobacter 43 (13.69\%), $\beta$-Hemolytic Streptococcus 15 (4.78\%), Pseudomonas species 111 (35.35\%), Streptococcus pneumoniae 10 (3.18\%) and Staphylococcus aureus in 17 cases (5.41\%). Gram-positive bacteria were isolated from 42 patients (13.51\%) while Gram-negative bacteria were isolated from 269 patients (86.49\%) (Table 2).

\begin{tabular}{|c|c|c|c|}
\hline S. No. & Name of Bacterium & $\begin{array}{l}\text { No. of } \\
\text { Isolates }\end{array}$ & Percentage \\
\hline \multicolumn{4}{|c|}{ Gram-positive } \\
\hline 1 & Streptococcus pneumoniae & 10 & $3.18 \%$ \\
\hline 2 & Staphylococcus aureus & 17 & $5.41 \%$ \\
\hline \multirow[t]{2}{*}{3} & B-Hemolytic Streptococcus & 15 & $4.78 \%$ \\
\hline & Total & 42 & $13.51 \%$ \\
\hline \multicolumn{4}{|c|}{ Gram-negative } \\
\hline 4 & Acinetobacter & 43 & $13.69 \%$ \\
\hline 5 & Pseudomonas aeruginosa & 111 & $35.35 \%$ \\
\hline 6 & Klebsiella species & 41 & $13.06 \%$ \\
\hline \multirow[t]{3}{*}{7} & Escherichia coli & 74 & $23.57 \%$ \\
\hline & Total & 269 & $86.49 \%$ \\
\hline & Grand Total & 311 & $100 \%$ \\
\hline
\end{tabular}

Table 2: Prevalence of respiratory tract bacteria.

Generally patients of age more than 50 years are more susceptible to respiratory tract infections due to less immunity. Among the patients of age group less than 15 years $\beta$-hemolytic Streptococcus has highest 
Citation: Ali I, Butt MA (2017) Antibiotic Susceptibility Pattern of Bacterial Isolates from Patients of Respiratory Tract Infection at 43 Centers in Punjab, Pakistan. Clin Exp Pharmacol 7: 229. doi:10.4172/2161-1459.1000229

Page 3 of 7

prevalence of $27 \%$ followed by Staphylococcus aureus (24\%). While among the patients of age group 15-25 years Escherichia coli has maximum prevalence of $20 \%$ followed by Pseudomonas aeruginosa (14\%). Among the patients of age group 25-50 years $\beta$-hemolytic
Streptococcus has highest prevalence of $47 \%$ followed by Pseudomonas aeruginosa. While among the patients of age group more than 50 years, Acinetobacter has maximum prevalence of $65 \%$ followed by Klebsiella which has prevalence of $63 \%$ (Table 3 ).

\begin{tabular}{|c|c|c|c|c|c|c|c|c|c|}
\hline \multirow{2}{*}{ S. No. } & \multirow{2}{*}{ Name of Bacterium } & \multicolumn{2}{|l|}{$<15$ Years } & \multicolumn{2}{|l|}{ 15-25 Years } & \multicolumn{2}{|l|}{ 25-50 Years } & \multicolumn{2}{|l|}{$>50$ Years } \\
\hline & & $\begin{array}{l}\text { No. of } \\
\text { Patients }\end{array}$ & $\begin{array}{l}\% \text { age of } \\
\text { Patients }\end{array}$ & $\begin{array}{l}\text { No. of } \\
\text { Patients }\end{array}$ & $\begin{array}{l}\% \text { age of } \\
\text { Patients }\end{array}$ & $\begin{array}{l}\text { No. of } \\
\text { Patients }\end{array}$ & $\begin{array}{l}\% \text { age of } \\
\text { Patients }\end{array}$ & $\begin{array}{l}\text { No. of } \\
\text { Patients }\end{array}$ & $\begin{array}{l}\% \text { age of } \\
\text { Patients }\end{array}$ \\
\hline 1 & Klebsiella species & 4 & $10 \%$ & 5 & $12 \%$ & 6 & $15 \%$ & 26 & $63 \%$ \\
\hline 2 & Escherichia coli & 13 & $18 \%$ & 15 & $20 \%$ & 18 & $24 \%$ & 28 & $38 \%$ \\
\hline 3 & Acinetobacter & -- & --- & 6 & $14 \%$ & 9 & $21 \%$ & 28 & $65 \%$ \\
\hline 4 & B-Hemolytic Streptococcus & 4 & $27 \%$ & 1 & $7 \%$ & 7 & $47 \%$ & 3 & $20 \%$ \\
\hline 5 & Pseudomonas aeruginosa & 8 & $7 \%$ & 16 & $14 \%$ & 36 & $32 \%$ & 51 & $46 \%$ \\
\hline 6 & $\begin{array}{l}\text { Streptococcus } \\
\text { pneumoniae }\end{array}$ & 1 & $10 \%$ & 1 & $10 \%$ & 3 & $30 \%$ & 5 & $50 \%$ \\
\hline \multirow[t]{2}{*}{7} & Staphylococcus aureus & 4 & $24 \%$ & 2 & $12 \%$ & 3 & $18 \%$ & 8 & $47 \%$ \\
\hline & Total & 34 & $11 \%$ & 46 & $15 \%$ & 82 & $26 \%$ & 149 & $48 \%$ \\
\hline
\end{tabular}

Table 3: Prevalence of respiratory bacteria in different age groups. On an average 30 antibiotics were tested against 7 isolated respiratory pathogens.

Klebsiella species showed maximum susceptibility to Cefoperazone/ Sulbactam and Carbapenems. $100 \%$ of Klebsiella species were susceptible to Cefoperazone/ Sulbactam and more than $91 \%$ to Imipenem and Meropenem. Escherichia coli showed the maximum susceptibility to Carbapenems, Cefoperazone/Sulbactam and Pipercillin/Tazobactam. More than $94 \%$ of Escherichia coli were susceptible to Imipenem, Meropenem and Cefoperazone/Sulbactam. However $89.7 \%$ of Escherichia coli were susceptible to Pipercillin/ Tazobactam. Acinetobacter showed maximum susceptibility to Cefoperazone/Sulbactam. $84.38 \%$ of Acinetobacter were susceptible to Cefoperazone/Sulbactam. $\beta$-hemolytic Streptococcus showed maximum susceptibility to Ampicillin, Amoxicillin, Amoxicillin/ Clavulanic acid, Ampicillin/Sulbactam and Ceftazidime. $100 \%$ of $\beta$ hemolytic Streptococcus was susceptible to Ampicillin, Amoxicillin, Amoxicillin/Clavulanic acid, Ampicillin/Sulbactam and Ceftazidime. However $91.67 \% \quad \beta$-hemolytic Streptococcus were susceptible to Doxycycline. Pseudomonas aeruginosa showed maximum susceptibility to Pipercillin/tazobactam, Cefoperazone/Sulbactam and Carbapenems.
More than $85 \%$ of Pseudomonas aeruginosa were susceptible to Pipercillin/tazobactam, Cefoperazone/Sulbactam and Imipenem. However $>80 \%$ of Pseudomonas aeruginosa were susceptible to Meropenem and Amikacin. Streptococcus pneumoniae showed maximum susceptibility to Penicillins, Cephalosporins, Carbapenems, Tigecycline and Vancomycin. 100\% of Streptococcus pneumoniae were susceptible to Penicillins, Cephalosporins, Carbapenems, Tigecycline and Vancomycin. However $83.33 \%$ of Streptococcus pneumoniae were susceptible to Doxycycline. Staphylococcus aureus showed maximum susceptibility to Vancomycin, Linezolid and Fusidic acid as $100 \%$, $92.31 \%$ and $83.33 \%$ respectively.

Twenty nine (29) antibiotics were tested against gram-positive respiratory pathogens. $66.25 \%$ of the antibiotics tested showed sensitivity to the gram-positive bacteria as shown in Table 4 . Vancomycin showed maximum sensitivity $(100 \%)$ to gram-positive bacteria followed by Linezolid (97.44\%) and Fusidic acid (83.34\%).

\begin{tabular}{|l|l|l|l|l|l|}
\hline S. No. & Name of Antibiotic & Staphylococcus aureus & $\begin{array}{l}\text { Streptococcus } \\
\text { pneumoniae }\end{array}$ & $\begin{array}{l}\boldsymbol{\beta} \text {-hemolytic } \\
\text { Streptococcus }\end{array}$ & Average percentage \\
\hline 1 & Vancomycin & $100.00 \%$ & $100.00 \%$ & $100.00 \%$ & $100.00 \%$ \\
\hline 2 & Linezolid & $92.31 \%$ & $100.00 \%$ & $100.00 \%$ & $97.44 \%$ \\
\hline 3 & Fusidic acid & $83.34 \%$ & $66.67 \%$ & $100.00 \%$ & $83.34 \%$ \\
\hline 4 & Cefipime & $46.15 \%$ & $100.00 \%$ & $100.00 \%$ & $82.05 \%$ \\
\hline 5 & Cefoperazone & $46.15 \%$ & $100.00 \%$ & $100.00 \%$ & $82.05 \%$ \\
\hline 6 & Cefotaxime & $46.15 \%$ & $100.00 \%$ & $100.00 \%$ & $82.05 \%$ \\
\hline
\end{tabular}


Citation: Ali I, Butt MA (2017) Antibiotic Susceptibility Pattern of Bacterial Isolates from Patients of Respiratory Tract Infection at 43 Centers in Punjab, Pakistan. Clin Exp Pharmacol 7: 229. doi:10.4172/2161-1459.1000229

Page 4 of 7

\begin{tabular}{|c|c|c|c|c|c|}
\hline 7 & Cefuroxime & $46.15 \%$ & $100.00 \%$ & $100.00 \%$ & $82.05 \%$ \\
\hline 8 & Amoxicillin/Clavulanic acid & $40.00 \%$ & $100.00 \%$ & $100.00 \%$ & $80.00 \%$ \\
\hline 9 & Ceftazidime & $40.00 \%$ & $100.00 \%$ & $100.00 \%$ & $80.00 \%$ \\
\hline 10 & Ceftriaxone & $40.00 \%$ & $100.00 \%$ & $100.00 \%$ & $80.00 \%$ \\
\hline 11 & Cefaclor & $40.00 \%$ & $100.00 \%$ & $100.00 \%$ & $80.00 \%$ \\
\hline 12 & Imipenem & $40.00 \%$ & $100.00 \%$ & $100.00 \%$ & $80.00 \%$ \\
\hline 13 & Meropenem & $40.00 \%$ & $100.00 \%$ & $100.00 \%$ & $80.00 \%$ \\
\hline 14 & Cephalexin & $40.00 \%$ & $100.00 \%$ & $80.00 \%$ & $73.33 \%$ \\
\hline 15 & Cephadrine & $40.00 \%$ & $100.00 \%$ & $80.00 \%$ & $73.33 \%$ \\
\hline 16 & Ampicillin/Sulbactam* & $16.67 \%$ & $100.00 \%$ & $100.00 \%$ & $72.22 \%$ \\
\hline 17 & Amoxicillin ${ }^{*}$ & $13.33 \%$ & $100.00 \%$ & $100.00 \%$ & $71.11 \%$ \\
\hline 18 & Ampicillin* & $13.33 \%$ & $100.00 \%$ & $100.00 \%$ & $71.11 \%$ \\
\hline 19 & Oxacillin & $30.77 \%$ & $100.00 \%$ & $66.67 \%$ & $65.81 \%$ \\
\hline 20 & Doxycycline & $20.00 \%$ & $83.33 \%$ & $91.67 \%$ & $65.00 \%$ \\
\hline 21 & Moxifloxacin & $46.15 \%$ & $25.00 \%$ & $75.00 \%$ & $48.72 \%$ \\
\hline 22 & Erythromycin & $30.77 \%$ & $75.00 \%$ & $38.46 \%$ & $48.08 \%$ \\
\hline 23 & Levofloxacin & $40.00 \%$ & $50.00 \%$ & $30.77 \%$ & $40.26 \%$ \\
\hline 24 & Ofloxacin & $40.00 \%$ & $50.00 \%$ & $30.76 \%$ & $40.25 \%$ \\
\hline 25 & Azithromycin ${ }^{*}$ & $0.00 \%$ & $75.00 \%$ & $38.46 \%$ & $37.82 \%$ \\
\hline 26 & Ciprofloxacin & $40.00 \%$ & $37.50 \%$ & $30.77 \%$ & $36.09 \%$ \\
\hline 27 & Gentamicin $^{*}$ & $40.00 \%$ & $0.00 \%$ & $66.67 \%$ & $35.56 \%$ \\
\hline 28 & Enoxacin ${ }^{*}$ & $33.33 \%$ & $50.00 \%$ & $11.11 \%$ & $31.48 \%$ \\
\hline \multirow[t]{2}{*}{29} & $\begin{array}{l}\text { Trimethoprim/ } \\
\text { Sulphamethoxazole }\end{array}$ & $33.33 \%$ & $25.00 \%$ & $7.69 \%$ & $22.01 \%$ \\
\hline & Average & $40.62 \%$ & $80.60 \%$ & $77.52 \%$ & $66.25 \%$ \\
\hline
\end{tabular}

Table 4: Anti-bacterial susceptibility of gram-positive bacteria. ${ }^{*}$ Antibiotic tested in less than $20 \%$ of either of Gram-positive isolates. All data is interpreted using (M100-S21 Vol. 31 No. 1) breakpoints.

Thirty four (34) antibiotics were tested against gram-negative respiratory pathogens. $33.44 \%$ of the antibiotics tested showed sensitivity to the gram-negative bacteria as shown in Table 5 .
Cefoperazone/Sulbactam showed maximum sensitivity (91.39\%) to gram-negative bacteria followed by Imipenem (72.75\%) and Pipercillin/Tazobactam (71.60\%).

\begin{tabular}{|c|c|c|c|c|c|c|}
\hline S. No. & Name of Antibiotic & Escherichia coli & $\begin{array}{l}\text { Pseudomonas } \\
\text { aeruginosa }\end{array}$ & $\begin{array}{l}\text { Klebsiella } \\
\text { species }\end{array}$ & Acinetobacter & $\begin{array}{l}\text { Average } \\
\text { percentage }\end{array}$ \\
\hline 1 & Cefoperazone/Sulbactam & $94.29 \%$ & $86.92 \%$ & $100.00 \%$ & $84.38 \%$ & $91.39 \%$ \\
\hline 2 & Imipenem ${ }^{*}$ & $95.71 \%$ & $86.11 \%$ & $92.11 \%$ & $17.07 \%$ & $72.75 \%$ \\
\hline 3 & Pipercillin/Tazobactam* & $89.71 \%$ & $89.90 \%$ & $97.05 \%$ & $9.76 \%$ & $71.60 \%$ \\
\hline 4 & Meropenem* & $95.71 \%$ & $82.08 \%$ & $91.43 \%$ & $17.07 \%$ & $71.57 \%$ \\
\hline 5 & Amikacin $^{*}$ & $76.06 \%$ & $80.00 \%$ & $97.37 \%$ & $9.76 \%$ & $65.80 \%$ \\
\hline
\end{tabular}


Citation: Ali I, Butt MA (2017) Antibiotic Susceptibility Pattern of Bacterial Isolates from Patients of Respiratory Tract Infection at 43 Centers in Punjab, Pakistan. Clin Exp Pharmacol 7: 229. doi:10.4172/2161-1459.1000229

Page 5 of 7

\begin{tabular}{|c|c|c|c|c|c|c|}
\hline 6 & Tobramycin* & $47.14 \%$ & $62.16 \%$ & $68.42 \%$ & $17.07 \%$ & $48.70 \%$ \\
\hline 7 & Gentamicin $^{*}$ & $47.89 \%$ & $66.67 \%$ & $73.68 \%$ & $2.50 \%$ & $47.68 \%$ \\
\hline 8 & Aztreonam* & $36.00 \%$ & $51.43 \%$ & $88.89 \%$ & $9.09 \%$ & $46.35 \%$ \\
\hline 9 & Cefipime* & $35.21 \%$ & $66.36 \%$ & $80.00 \%$ & $0.00 \%$ & $45.39 \%$ \\
\hline 10 & Ciprofloxacin ${ }^{*}$ & $33.80 \%$ & $57.66 \%$ & $71.05 \%$ & $2.44 \%$ & $41.24 \%$ \\
\hline 11 & Levofloxacin ${ }^{*}$ & $30.99 \%$ & $51.40 \%$ & $71.43 \%$ & $9.76 \%$ & $40.89 \%$ \\
\hline 12 & Moxifloxacin ${ }^{*}$ & $32.84 \%$ & $53.09 \%$ & $71.42 \%$ & $3.13 \%$ & $40.12 \%$ \\
\hline 13 & Cefoperazone $^{*}$ & $28.79 \%$ & $55.85 \%$ & $71.05 \%$ & $0.00 \%$ & $38.92 \%$ \\
\hline 14 & Ofloxacin & $28.57 \%$ & $50.45 \%$ & $71.05 \%$ & $4.88 \%$ & $38.74 \%$ \\
\hline 15 & Ampicillin/Sulbactam* & $22.22 \%$ & $25.00 \%$ & $100.00 \%$ & $0.00 \%$ & $36.81 \%$ \\
\hline 16 & Ceftazidime & $29.58 \%$ & $29.73 \%$ & $71.05 \%$ & $2.44 \%$ & $33.20 \%$ \\
\hline 17 & Cefotaxime $^{*}$ & $26.76 \%$ & $29.09 \%$ & $71.05 \%$ & $0.00 \%$ & $31.73 \%$ \\
\hline 18 & Enoxacin* & $0.00 \%$ & $24.00 \%$ & $100.00 \%$ & $0.00 \%$ & $31.00 \%$ \\
\hline 19 & Cefixime $^{*}$ & $0.00 \%$ & $50.00 \%$ & $71.43 \%$ & $0.00 \%$ & $30.36 \%$ \\
\hline 20 & Ceftriaxone $^{*}$ & $25.35 \%$ & $20.91 \%$ & $71.05 \%$ & $0.00 \%$ & $29.33 \%$ \\
\hline 21 & Amoxicillin* & $12.50 \%$ & $100.00 \%$ & $0.00 \%$ & $0.00 \%$ & $28.13 \%$ \\
\hline 22 & Nitrofurantoin ${ }^{*}$ & $100.00 \%$ & $0.00 \%$ & $0.00 \%$ & $0.00 \%$ & $25.00 \%$ \\
\hline 23 & Doxycycline* & $12.86 \%$ & $12.05 \%$ & $32.43 \%$ & $39.47 \%$ & $24.20 \%$ \\
\hline 24 & Cefuroxime* $^{*}$ & $19.72 \%$ & $6.54 \%$ & $67.57 \%$ & $0.00 \%$ & $23.46 \%$ \\
\hline 25 & $\begin{array}{l}\text { Trimethoprim/ } \\
\text { Sulphamethoxazole }\end{array}$ & $20.00 \%$ & $14.41 \%$ & $42.86 \%$ & $0.00 \%$ & $19.32 \%$ \\
\hline 26 & Amoxicillin/Clavulanic acid ${ }^{*}$ & $15.49 \%$ & $12.50 \%$ & $47.36 \%$ & $0.00 \%$ & $18.84 \%$ \\
\hline 27 & Cefaclor ${ }^{*}$ & $11.27 \%$ & $2.44 \%$ & $54.05 \%$ & $0.00 \%$ & $16.94 \%$ \\
\hline 28 & Cephalexin* & $8.45 \%$ & $2.22 \%$ & $30.56 \%$ & $0.00 \%$ & $10.31 \%$ \\
\hline 29 & Cephadrine* $^{*}$ & $8.45 \%$ & $2.35 \%$ & $25.00 \%$ & $0.00 \%$ & $8.95 \%$ \\
\hline 30 & Ticarcilin/Clavulanic acid & $0.00 \%$ & $20.00 \%$ & $0.00 \%$ & $0.00 \%$ & $5.00 \%$ \\
\hline 31 & Ampicillin* & $12.50 \%$ & $0.00 \%$ & $0.00 \%$ & $0.00 \%$ & $3.13 \%$ \\
\hline 32 & Minocyclin* & $0.00 \%$ & $0.00 \%$ & $0.00 \%$ & $0.00 \%$ & $0.00 \%$ \\
\hline 33 & Naladixic acid ${ }^{*}$ & $0.00 \%$ & $0.00 \%$ & $0.00 \%$ & $0.00 \%$ & $0.00 \%$ \\
\hline \multirow[t]{2}{*}{34} & Pipemedic Acid ${ }^{*}$ & $0.00 \%$ & $0.00 \%$ & $0.00 \%$ & $0.00 \%$ & $0.00 \%$ \\
\hline & Average & $32.29 \%$ & $37.98 \%$ & $56.75 \%$ & $6.73 \%$ & $33.44 \%$ \\
\hline
\end{tabular}

Table 5: Anti-bacterial susceptibility of gram-negative bacteria. ${ }^{*}$ Antibiotic tested in less than $20 \%$ of either of Gram-negative isolates. All data is interpreted using (M100-S21 Vol. 31 No. 1) breakpoints.

Overall $42.23 \%$ of the bacterial isolates showed susceptibility to the antibiotics tested as shown in Table 6. Imipenem was tested against more than $25 \%$ of each of gram-positive and gram-negative isolates. Bacterial isolates showed highest susceptibility of $76.38 \%$ to Imipenem. Susceptibility to Meropenem, Cefipime was $75.79 \%$ and $63.72 \%$ respectively. Among orally available antibiotics, susceptibility to Moxifloxacin was $57.56 \%$, but as the prevalence of gram-negative bacteria is more than gram-positive bacteria, Cefuroxime $(52.75 \%)$ is best option for empiric therapy in respiratory tract infection which is cheaper than Moxifloxacin. 
Citation: Ali I, Butt MA (2017) Antibiotic Susceptibility Pattern of Bacterial Isolates from Patients of Respiratory Tract Infection at 43 Centers in Punjab, Pakistan. Clin Exp Pharmacol 7: 229. doi:10.4172/2161-1459.1000229

Page 6 of 7

\begin{tabular}{|c|c|c|c|c|}
\hline \multicolumn{4}{|c|}{ Antibacterial Susceptibility Pattern } & \multirow[b]{2}{*}{$\begin{array}{l}\text { Percentage Sensitivity of } \\
\text { all Isolates }\end{array}$} \\
\hline S. No. & Name of Antibiotics & $\begin{array}{l}\text { Percentage Sensitivity of Gram- } \\
\text { negative Isolates }\end{array}$ & $\begin{array}{l}\text { Percentage Sensitivity of Gram- } \\
\text { positive Isolates }\end{array}$ & \\
\hline 1 & Imipenem & $72.75 \%$ & $80.00 \%$ & $76.38 \%$ \\
\hline 2 & Meropenem & $71.57 \%$ & $80.00 \%$ & $75.79 \%$ \\
\hline 3 & Cefipime & $45.39 \%$ & $82.05 \%$ & $63.72 \%$ \\
\hline 4 & Cefoperazone & $38.92 \%$ & $82.05 \%$ & $60.49 \%$ \\
\hline 5 & Doxycycline & $24.20 \%$ & $91.67 \%$ & $57.93 \%$ \\
\hline 6 & Moxifloxacin & $40.12 \%$ & $75.00 \%$ & $57.56 \%$ \\
\hline 7 & Gentamicin & $47.68 \%$ & $66.67 \%$ & $57.18 \%$ \\
\hline 8 & Cefotaxime & $31.73 \%$ & $82.05 \%$ & $56.89 \%$ \\
\hline 9 & Ceftazidime & $33.20 \%$ & $80.00 \%$ & $56.60 \%$ \\
\hline 10 & Ceftriaxone & $29.33 \%$ & $80.00 \%$ & $54.66 \%$ \\
\hline 11 & Ampicillin/Sulbactam & $36.81 \%$ & $72.22 \%$ & $54.51 \%$ \\
\hline 12 & Cefuroxime & $23.46 \%$ & $82.05 \%$ & $52.75 \%$ \\
\hline 13 & Vancomycin* & $0.00 \%$ & $100.00 \%$ & $50.00 \%$ \\
\hline 14 & Linezolid* & $0.00 \%$ & $100.00 \%$ & $50.00 \%$ \\
\hline 15 & Fusidic Acid ${ }^{*}$ & $0.00 \%$ & $100.00 \%$ & $50.00 \%$ \\
\hline 16 & Amoxicillin & $28.13 \%$ & $71.11 \%$ & $49.62 \%$ \\
\hline 17 & Amoxicillin/Clavulanic acid* & $18.84 \%$ & $80.00 \%$ & $49.42 \%$ \\
\hline 18 & Cefaclor* & $16.94 \%$ & $80.00 \%$ & $48.47 \%$ \\
\hline 19 & Cefoperazone/Sulbactam & $91.39 \%$ & $0.00 \%$ & $45.70 \%$ \\
\hline 20 & Cephalexin* & $10.31 \%$ & $73.33 \%$ & $41.82 \%$ \\
\hline 21 & Cephadrine $^{*}$ & $8.95 \%$ & $73.33 \%$ & $41.14 \%$ \\
\hline 22 & Ampicillin* & $3.13 \%$ & $71.11 \%$ & $37.12 \%$ \\
\hline 23 & Ciprofloxacin & $41.24 \%$ & $30.77 \%$ & $36.00 \%$ \\
\hline 24 & Levofloxacin & $40.89 \%$ & $30.77 \%$ & $35.83 \%$ \\
\hline 25 & Pipercillin/Tazobactam* & $71.60 \%$ & $0.00 \%$ & $35.80 \%$ \\
\hline 26 & Ofloxacin & $38.74 \%$ & $30.76 \%$ & $34.75 \%$ \\
\hline 27 & Oxacillin* & $0.00 \%$ & $65.81 \%$ & $32.91 \%$ \\
\hline 28 & Amikacin* & $65.80 \%$ & $0.00 \%$ & $32.90 \%$ \\
\hline 29 & Tobramycin* & $48.70 \%$ & $0.00 \%$ & $24.35 \%$ \\
\hline 30 & Aztreonam* & $46.35 \%$ & $0.00 \%$ & $23.18 \%$ \\
\hline 31 & Enoxacin* & $31.00 \%$ & $11.11 \%$ & $21.06 \%$ \\
\hline 32 & Azithromycin* & $0.00 \%$ & $38.46 \%$ & $19.23 \%$ \\
\hline 33 & Erythromycin* & $0.00 \%$ & $38.46 \%$ & $19.23 \%$ \\
\hline 34 & Cefixime* $^{*}$ & $30.36 \%$ & $0.00 \%$ & $15.18 \%$ \\
\hline
\end{tabular}


Citation: Ali I, Butt MA (2017) Antibiotic Susceptibility Pattern of Bacterial Isolates from Patients of Respiratory Tract Infection at 43 Centers in Punjab, Pakistan. Clin Exp Pharmacol 7: 229. doi:10.4172/2161-1459.1000229

Page 7 of 7

\begin{tabular}{|l|l|l|l|l|}
\hline 35 & Trimethoprim/Sulphamethoxazole* & $19.32 \%$ & $7.69 \%$ & $13.50 \%$ \\
\hline 36 & Nitrofurantoin* & $25.00 \%$ & $0.00 \%$ & $12.50 \%$ \\
\hline 37 & Ticarcilin/Clavulanic acid* & $5.00 \%$ & $0.00 \%$ & $2.50 \%$ \\
\hline & Total & $31.58 \%$ & $52.88 \%$ & $42.23 \%$ \\
\hline
\end{tabular}

Table 6: Anti-bacterial susceptibility pattern. ${ }^{*}$ Antibiotic tested in less than $20 \%$ of either of Gram-positive and Gram-negative isolates. All data is interpreted using CLSI (M100-S21 Vol. 31 No. 1) breakpoints.

\section{Discussion}

Resistance is an extremely scary situation for both patients and health care workers. Drug side effects, allergic reactions and diarrheal infections result from inappropriate use of antibiotics. There is need of surveillance studies of antibiotic susceptibility at national level at regular intervals. Physician should be well aware of susceptibility patterns in a particular region before prescribing antibiotics. Empiric therapy should be based on most recent study.

The present study showed that certain antibiotics were more effective and cheaper than other antibiotics, e.g., in Streptococcus pneumonia, Amoxicillin and Ampicillin are effective and more economical than Vancomycin, Linezolid and Tigecycline.

Health care providers, hospital administrators and policy maker must work together in reducing unnecessary antibiotic use, emphasis and implement intervention system. Government of Pakistan should review their policies regarding rational use of antibiotics and restrict over the counter sale of antibiotics. Hygenicity and sterility of medical equipment should not be compromised at any cost.

From the data, the antibiotics having low susceptibility profile (i.e. Trimethoprim/Sulphamethoxazole) should not be prescribed (in empiric therapy) and dispensed; further the manufacturing should be hold for ample period of time to regain its susceptibility back. On the other side, the prescriber should prefer the antibiotics with good susceptibility profile (i.e., Imipenem).

\section{Conclusion}

This study was conducted to ascertain the current scenario of bacterial susceptibility in respiratory tract infection to optimize empiric therapy among people of all groups of ages in Punjab province located in Pakistan.

The present study concluded that Imipenem which is a broad spectrum antibiotic is most suitable as an empirical therapy in respiratory tract infections in hospital setting followed by Meropenem and in general practice Doxycycline is most effective as oral antibiotic followed by Moxifloxacin.

\section{References}

1. Stefani S (2000) Changes in the resistance patterns among upper respiratory tract infection pathogens. Int J Antimicrob Agents 16: 493-494.

2. Lui KJ, Kendal AP (1987) Impact of influenza epidemics on mortality in the United States from October 1972 to May 1985. Am J Public Health 77: 712-716.

3. Gelone SP, O'Donnell J (2008) Chapter 60 Respiratory Tract Infections. In: Koda-Kimble MA, Young LY, Kradja WA, Guglielmo BJ, Alldredge BK, et al. (eds.) Applied Therapeutics: The Clinical Use of Drugs Point. Lippincott Williams \& Wilkins.

4. Dixon RE (1985) Economic costs of respiratory tract infections in the United States. Am J Med 78: 45-51.

5. National Institute of Health and World Health Organization (WHO) (2011) Weekly Epidemological Bulletin, Disease Early Warning System and response in Pakistan 2: 4.

6. Rang HP, Dale MM, Ritter JM, Flower RJ, Henderson G (2011) Rang \& Dale's Pharmacology (Elsevier Health Sciences).

7. Bambeke FV, Pages JM, Lee VJ (2006) Inhibitors of bacterial efflux pumps as adjuvants in antibiotic treatments and diagnostic tools for detection of resistance by efflux. Recent Pat Antiinfect Drug Discov 1: 157-175.

8. De LH, Oliveria D, Tomasz A (2007) Antibiotic resistant Staphylococcus aureus. a paradigm of adaptive power. Curr Opin Microbiol 10: 428-435.

9. Barrett CT, Barrett JF (2003) Antibacterials: are the new entries enough to deal with the emerging resistance problem? Curr Opin Biotechnol 14: 621-626.

10. Lesar TS, Rotschafer JC, Strand LM, Solem LD, Zaske DE (1982) Gentamicin dosing errors with four commonly used nomograms. JAMA 248: 1190-1193.

11. Cheesbrough M (1999) District Laboratory Practice in Tropical Countries. Cambridge University Press. 